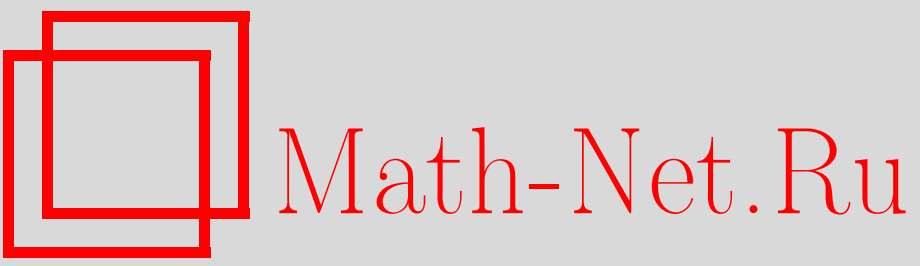

Н. А. Кудряшов, Обобщения уравнений Пенлеве, ТМФ, 2003, том 137, номер 3, 408-423

DOI: https://doi.org/10.4213/tmf281

Использование Общероссийского математического портала Math-Net.Ru подразумевает, что вы прочитали и согласны с пользовательским соглашением

http://www . mathnet.ru/rus/agreement

Параметры загрузки:

IP : 54.162 .27 .143

26 апреля 2023 г., 10:07:54 


\author{
ТЕОРЕТИЧЕСКАЯ \\ И МАТЕМАТИЧЕСКАЯ \\ ФИЗИКА \\ Том 137, № 3 \\ декабрь, 2003
}

(C) 2003 г.

\title{
ОБОБЩЕНИЯ УРАВНЕНИЙ ПЕНЛЕВЕ
}

Представлены новые иерархии нелинейных обыкновенных дифференциальных уравнений, являющиеся обобщениями уравнений Пенлеве. Показано, что эти иерархии содержат уравнения Пенлеве как частные случаи. Особое внимание уделено обыкновенным дифференциальным уравнениям шестого порядка. Частные решения одного из них выражаются через общие решения $P_{1}-, P_{2}$-уравнений и частные случаи $P_{3}$ - и $P_{5}$-уравнений. Четыре из шести уравнений Пенлеве можно рассматривать как частные случаи этого обыкновенного дифференциального уравнения шестого порядка. Приведены линейные представления для решения задач Коши для уравнений иерархий с помощью обратного преобразования монодромии.

Ключевые слова: уравнения Пенлеве, трансценденты Пенлеве, высшие аналоги, изомонодромийная линейная задача.

\section{1. ВВЕДЕНИЕ}

Как хорошо известно, более века назад Пенлеве и его ученики открыли шесть обыкновенных дифференциальных уравнений (ОДУ), определяющих новые существенно трансцендентные функции относительно постоянных интегрирования. Это было достигнуто путем классификации ОДУ второго порядка некоторого вида, обладающих тем, что в настояшее время называют свойством Пенлеве (обшее решение не имеет подвижных критических точек).

Пенлеве и его сотрудники нашли пятьдесят канонических уравнений, решения которых не имеют подвижных критических точек. Более того, они также показали, что среди этих пятидесяти уравнений имеются ровно шесть обыкновенных дифференциальных уравнений второго порядка, определяюших новые функции. В настояшее время эти новые функции называются трансцендентами Пенлеве, а уравнения с общими решениями в виде этих трансцендентов называются уравнениями Пенлеве. Эти шесть уравнений Пенлеве были впервые обнаружены в ходе чисто математических исследований, однако недавно они возникли и в некоторых физических приложениях [1].

Результаты Пенлеве и его школы привели к необходимости решения таких задач, как классификация других типов нелинейных дифференциальных уравнений и поиск

* Московский инженерно-физический институт (государственный университет), Москва, Россия. E-mail: kudr@dampe.mephi.ru 
дифференциальных уравнений высших порядков, определяющих новые трансцендентные функции по отношению к постоянным интегрирования. Недавно была предпринята попытка найти новые функции, отличные от трансцендентов Пенлеве, также определяемые нелинейными ОДУ. Для этой цели были введены несколько иерархий ОДУ с использованием иерархий нелинейных дифференциальных уравнений в частных производных (ДУЧП), решаемых с помошью метода обратной задачи рассеяния [2]-[5].

Цель настоящей работы - представить новые иерархии ОДУ, которые имеют свойства, аналогичные уравнениям Пенлеве. Особенность, которая выделяет эти иерархии, состоит в том, что они содержат ряд уравнений Пенлеве в качестве частного случая.

Работа построена следуюшим образом. Метод нахождения новых иерархий для обобшения уравнений Пенлеве представлен в разделе 2. Иерархии с линейным потенциалом рассматриваются в разделе 3 .

\section{2. ИСПОЛЬЗОВАННЫЙ МЕТОД}

Известно, что уравнения Пенлеве можно записать как изомонодромийные линейные задачи. Гарнье и Фукс первыми [6] нашли, что пять из шести уравнений Пенлеве можно представить как изомонодромийные линейные задачи, которые можно использовать для решения задач Коши для уравнений Пенлеве с помошью обратного преобразования монодромии [7], [8].

Для того чтобы решить задачи Коши для $P_{1}, P_{34}$ и для частных случаев $P_{3}$ - и $P_{5}$-уравнений, линейные задачи можно представить в виде системы уравнений [9]

$$
\begin{gathered}
\Psi_{x x}=(P(x)-\lambda) \Psi, \\
\omega(\lambda) \Psi_{\lambda}=2 A(x, \lambda) \Psi_{x}-A_{x}(x, \lambda) \Psi,
\end{gathered}
$$

где $A(x, \lambda)$ принимает вид

$$
A(x, \lambda)=a_{1}(x)+a_{0}(x) \lambda .
$$

Если один из параметров третьего (пятого) уравнения Пенлеве равен нулю, мы говорим, что имеется частный случай третьего (пятого) уравнения Пенлеве. Далее будем обозначать эти частные случаи уравнений как $P_{3}^{*}$ - и $P_{5}^{*}$-уравнения.

Условие совместности для системы уравнений (2.1)

$$
\left(\Psi_{x x}\right)_{\lambda}=\left(\Psi_{\lambda}\right)_{x x}
$$

можно записать в виде следующего уравнения [10], [11]:

$$
\omega U_{\lambda}=4 U A_{x}+2 U_{x} A-A_{x x x},
$$

где мы использовали потенциал $U(x, \lambda)$ в линейной форме:

$$
U(x, \lambda)=P(x)-\lambda .
$$

Предполагая (2.2) и полагая $\omega(\lambda)=1$ в системе уравнений $(2.1)$, получаем линейную задачу для первого уравнения Пенлеве. При $\omega(\lambda)=\lambda$ получаем пару Лакса для 
$P_{34}$-уравнения. Учитывая (2.2), а также $\omega(\lambda)=\lambda^{2}$ и $\omega(\lambda)=\lambda\left(\lambda-\lambda_{0}\right)$, получаем линейные задачи для $P_{3}^{*}$ - и $P_{5}^{*}$-уравнений [10].

Условие совместности (2.3) изомонодромийной линейной задачи вида

$$
\begin{gathered}
\Psi_{x x}=2(\lambda-y) \Psi_{x}+Q(x) \Psi, \\
\omega(\lambda) \Psi_{\lambda}=C(x, \lambda) \Psi_{x}+D(x, \lambda) \Psi
\end{gathered}
$$

также можно записать как уравнение (2.4), если положить

$$
\begin{gathered}
Q(x)=P(x)-y_{x}-y^{2}, \quad C(x, \lambda)=2 A(x, \lambda), \\
D(x, \lambda)=2 A_{x}(x, \lambda)(y-\lambda)+\omega(\lambda) x-A_{x}(x, \lambda), \\
U(x, \lambda)=P(x)-2 \lambda y(x)+\lambda^{2} .
\end{gathered}
$$

Предполагая (2.2) и полагая $\omega(\lambda)=1$ в системе уравнений (2.6), мы получаем пару Лакса для второго уравнения Пенлеве. В случае уравнения $(2.2)$ при $\omega(\lambda)=\lambda$ получаем линейную задачу для четвертого уравнения Пенлеве. Учитьвая $(2.2)$ и $\omega(\lambda)=\lambda^{2}$, получаем пару Лакса для третьего уравнения Пенлеве. В случае $(2.2)$ при $\omega(\lambda)=\lambda\left(\lambda-\lambda_{0}\right)$ имеем изомонодромийную линейную задачу для пятого уравнения Пенлеве. Потеншиал (2.9) также рассматривался в работе [12].

Уравнение (2.4) можно записать следуюшим образом:

$$
\frac{\partial}{\partial x}\left(2 U A^{2}-A A_{x x}+\frac{1}{2} A_{x}^{2}\right)=\omega U_{\lambda} A .
$$

Из (2.10) можно видеть, что сушествует интеграл этого уравнения при $\omega(\lambda)=0$ вида

$$
2 U A^{2}-A A_{x x}+\frac{1}{2} A_{x}^{2}=\text { const } .
$$

Уравнение (2.10) позволяет также искать интегралы уравнения $(2.4)$, когда $\omega(\lambda)$ имеет корни. Мы используем эти уравнения в разделе 3 настоящей работы.

Используя условие совместности $(2.3)$ и $A(x, \lambda)$ в виде

$$
A(x, \lambda)=a_{2}(x)+a_{1}(x) \lambda+a_{0}(x) \lambda^{2},
$$

мы нашли в нашей недавней работе [5] несколько новых ОДУ четвертого порядка, которые являются аналогами уравнений Пенлеве.

Полагая, что

$$
A(x, \lambda)=\sum_{i=0}^{n} a_{i}(x) \lambda^{n-i},
$$

ниже мы найдем несколько новых иерархий ОДУ со свойствами, аналогичными уравнениям Пенлеве. 


\section{3. ИЕРАРХИИ С ЛИНЕЙНЫМ ПОТЕНЦИАЛОМ (2.5)}

Для того чтобы найти иерархии, являюшиеся обобшениями уравнений Пенлеве, мы будем исследовать уравнение (2.4). В первую очередь используем линейный потенциал (2.5) в уравнении (2.4).

3.1. Случай $\omega(\lambda)=\omega_{0}=$ const. Подставляя в $(2.4) U(x, \lambda)$ в виде $(2.5)$ и $\omega(\lambda)=\omega_{0}$, получим

$$
4 P A_{x}+2 P_{x} A-A_{x x x}+\omega_{0}-4 \lambda A_{x}=0 .
$$

Полагая, что $A(x, \lambda)$ выражается с помошью формулы (2.13), и приравнивая нулю выражения при различных $\lambda$, получим

$$
\begin{gathered}
a_{0, x}=0, \\
4 a_{k+1, x}=4 P a_{k, x}+2 P_{x} a_{k}-a_{k, x x x}, \quad k=1, \ldots, n-1, \\
4 P a_{n, x}+2 P_{x} a_{n, x}-a_{n, x x x}+\omega_{0}=0 .
\end{gathered}
$$

Уравнения (3.2)-(3.4) можно проинтегрировать по $x$. В результате получаем

$$
\begin{gathered}
a_{0}(x)=c_{0}, \\
a_{1}(x)=\frac{1}{2} c_{0} P(x)+c_{1}, \\
a_{2}(x)=-\frac{c_{0}}{8}\left(P_{x x}-3 P^{2}\right)+\frac{1}{2} c_{1} P+c_{2} .
\end{gathered}
$$

Можно видеть, что последний коэффишиент записывается в виде

$$
a_{2}(x)=-\frac{c_{0}}{8} L^{2}[P]+\frac{1}{2} c_{1} L^{1}[P]+c_{2},
$$

где оператор $L^{k}[P]$ определен с помошью соотношения Ленарда [13]:

$$
\frac{d}{d x} L^{k+1}[P]=-4 P L_{x}^{k}[P]-2 P_{x} L^{k}[P]+L_{x x x}^{k}[P], \quad L^{1}[P]=P .
$$

Теперь можно сформулировать следующую теорему.

Теорема 3.1. Коәффициенты $a_{m}(x), m=1, \ldots, n$, являюощиеся решениями уравнений (3.2) и (3.3), определяются формулой

$$
a_{m}(x)=\frac{1}{2} \sum_{i=1}^{m}\left(-\frac{1}{4}\right)^{i-1} c_{m-i} L^{i}[P]+c_{m}, \quad m=1, \ldots, n .
$$


ДокАЗАТЕЛЬСТво. Эта теорема доказывается методом математической индукции. При $m=1$ и $m=2$ уравнение (3.10) сводится к уравнениям (3.6) и (3.7). Полагая $m=p$ в (3.10), получаем решение уравнения (3.3) при $m=p+1$.

Подставляя $a_{m}(x)$ при $m=n$ в уравнение (3.4), после интегрирования получаем следуюшее уравнение:

$$
\frac{1}{2} \sum_{i=1}^{n+1}\left(-\frac{1}{4}\right)^{i-1} c_{n+1-i} L^{i}[P]+c_{n+1}=-\omega_{0} x
$$

Обозначая

$$
P(x)=y(x), \quad \omega_{0}=-\alpha, \quad \alpha_{i}=\frac{1}{2}\left(-\frac{1}{4}\right)^{i-1} c_{n+1-i}, \quad i=1, \ldots, n+1,
$$

и меняя переменные $x$ и $y$, получаем уравнение вида

$$
\sum_{i=2}^{n+1} \alpha_{i} L^{i}[y]=\alpha x
$$

Эта иерархия является обобшением первой иерархии Пенлеве, которая была найдена в работе [3] как редукция иерархии Шварца-Кортевега-де Фриза с использованием самоподобных решений. Иерархия (3.12) эквивалентна также обобшенной $P_{1}$-иерархии, приведенной в [14].

Полагая $\alpha_{i \neq 2}=0, \alpha_{2}=1$, получаем первое уравнение Пенлеве. В случае $\alpha_{i}=0$ $(i \neq 1,2), \alpha_{2}=\beta, \alpha_{1}=1$ получаем нелинейное ОДУ четвертого порядка, которое было найдено в недавних работах [5], [15]-[17]. Некоторые свойства этого уравнения при $\alpha_{4}=1, \alpha_{i \neq 4}=0$ были изучены в работах [14], [18], [19]. Случай уравнения четвертого порядка для уравнения (3.12) также обсуждался в [11].

При $n=3$ уравнение иерархии (3.12) принимает вид

$$
\begin{aligned}
& \alpha_{4}\left(y_{x x x x x x}-35 y^{4}-21 y_{x x}^{2}-14 y y_{x x x x}-28 y_{x} y_{x x x}+70 y^{2} y_{x x}+70 y y_{x}^{2}\right)+ \\
& \quad+\alpha_{3}\left(y_{x x x x}-5 y_{x}^{2}-10 y y_{x x}+10 y^{3}\right)+\alpha_{2}\left(y_{x x}-3 y^{2}\right)=\alpha x
\end{aligned}
$$

Уравнение (3.13) при $\alpha_{4}=0$ также было найдено в работе [16]. Изомонодромийная линейная задача, соответствуюшая уравнению (3.12), выражается системой уравнений $(2.1)$, где $A(x, \lambda)$ определяется в $(2.13)$, а коэффициенты $a_{i}(x)$ - по формуле $(3.10)$.

3.2. Случай $\omega(\lambda)=\omega_{0} \lambda, \omega_{0}=$ const. Используя $U(x, \lambda)$ в виде линейного потенциала $(2.5)$ и соотношение $\omega(\lambda)=\omega_{0} \lambda$, из уравнения $(2.4)$ получаем

$$
4 P A_{x}+2 P_{x} A-A_{x x x}+\omega_{0} \lambda-4 \lambda A_{x}=0 .
$$


Подставляя $A(x, \lambda)$ в виде $(2.13)$ в (3.14) и приравнивая нулю выражения при различных $\lambda$, получаем

$$
\begin{gathered}
a_{0, x}=0, \\
4 a_{k+1, x}=4 P a_{k, x}+2 P_{x} a_{k}-a_{k, x x x}, \quad k=1, \ldots, n-2, \\
4 P a_{n-1, x}+2 P_{x} a_{n-1}-a_{n-1, x x x}-4 a_{n, x}+\omega_{0}=0, \\
4 P a_{n, x} a_{n}+2 P_{x} a_{n, x}-a_{n, x x x}=0 .
\end{gathered}
$$

Из (3.14) и (3.15) при $k \leqslant n-2$ следуют те же уравнения, что и в предыдущем случае. Подставляя (3.10) в (3.16) при $m=1, \ldots, n-2$, получаем

$$
a_{n-1}(x)=\frac{1}{2} \sum_{i=1}^{n-1}\left(-\frac{1}{4}\right)^{i-1} c_{n-1-i} L^{i}[P]+c_{n-1} .
$$

Подставляя $(3.19)$ в (3.17) и обозначая

$$
P(x)=y(x), \quad \alpha_{k}=\frac{1}{2}\left(-\frac{1}{4}\right)^{k-1} c_{n-k}, \quad k=1, \ldots, n, \quad a_{n}(x)=u(x), \quad \omega_{0}=-\alpha
$$

получаем систему уравнений вида

$$
\begin{gathered}
\sum_{k=1}^{n} \alpha_{k} L^{k}[y]+4 u(x)=\alpha x, \\
2 y u^{2}-u u_{x x}+\frac{1}{2} u_{x}^{2}-\frac{\delta^{2}}{2}=0 .
\end{gathered}
$$

Полагая $u(x)=\delta=0$, из уравнений (3.20) и (3.21) получаем иерархию (3.12). В случае $\alpha_{k}=0$ при $k \neq 1, \alpha_{1}=1$ находим

$$
u u_{x x}-\frac{1}{2} u_{x}^{2}-2 u^{2} \alpha x+8 u^{3}+\frac{\delta^{2}}{2}=0 .
$$

Это уравнение представляет собой $P_{34}$-уравнение. Его можно преобразовать ко второму уравнению Пенлеве, если воспользоваться переменными вида

$$
S(x)=\frac{u_{x}+\delta}{2 u} .
$$

В результате получаем второе уравнение Пенлеве в виде

$$
S_{x x}=2 S^{3}-2 \alpha x S+4 \delta+\alpha .
$$

Полагая $n=3$ в системе уравнений (3.20) и (3.21), получаем

$$
\begin{gathered}
\alpha_{3}\left(y_{x x x x}-5 y_{x}^{2}-10 y y_{x x}+10 y^{3}\right)+\alpha_{2}\left(y_{x x}-3 y^{2}\right)+\alpha_{1} y+4 u=\alpha x \\
u u_{x x}-\frac{1}{2} u_{x}^{2}-2 y u^{2}+\frac{\delta^{2}}{2}=0 .
\end{gathered}
$$


Иерархию (3.20) и (3.21) можно рассматривать как обобшение первой иерархии Пенлеве и второго уравнения Пенлеве.

Подставляя (3.23) в уравнение $(3.21)$, находим

$$
y=S_{x}+S^{2}
$$

В этом случае уравнения (3.20) можно представить в виде

$$
\sum_{k=1}^{n} \alpha_{k} L^{k}\left[S_{x}+S^{2}\right]+4 u=\alpha x
$$

Принимая во внимание уравнения (3.23) и (3.28), получаем обобшение второй иерархии Пенлеве в виде

$$
\left(\frac{d}{d x}-2 S\right) \sum_{k=1}^{n} \alpha_{k} L^{k}\left[S_{x}+S^{2}\right]+2 \alpha x S-\alpha-4 \delta=0 .
$$

Эта иерархия эквивалентна обобшенной $P_{2}$-иерархии, которая приведена в работе [14]. Изомонодромийная линейная задача для иерархии (3.20) и (3.21) выражается системой уравнений $(2.1)$, где $U(x, \lambda)$ есть уравнение $(2.5)$, а $A(x, \lambda)-(2.13)$.

Иерархия (3.29) является обобшением второй иерархии Пенлеве [2], [3], [7], [20]. Уравнения иерархии (3.29) будут иметь частные и рациональные решения.

Можно видеть, что при $n=1$ уравнение (3.56) является вторым уравнением Пенлеве. В случае $n=2$ уравнение (3.29) можно представить в виде

$$
\begin{aligned}
& \alpha_{2}\left(S_{x x x x}-10 S S_{x}^{2}-10 S^{2} S_{x x}+6 S^{5}\right)+ \\
& \quad+\alpha_{1}\left(S_{x x}-2 S^{3}\right)+2 \alpha x S-\alpha-4 \delta=0 .
\end{aligned}
$$

Это уравнение было найдено в недавней работе [21]. Можно видеть, что при $\alpha_{2}=$ 0 оно сводится ко второму уравнению Пенлеве, однако при $\alpha_{1}=0$ становится вторым членом $P_{2}$-иерархии.

При $n=3$ из уравнения (3.29) имеем уравнение шестого порядка вида

$$
\begin{gathered}
\alpha_{3}\left(S_{x x x x x x}-56 S S_{x} S_{x x x}-14 S^{2} S_{x x x x}-42 S S_{x x}^{2}-\right. \\
\left.-70 S_{x}^{2} S_{x x}+70 S^{4} S_{x x}+140 S_{x}^{2} S^{3}-20 S^{7}\right)+ \\
+\alpha_{2}\left(S_{x x x x}-10 S S_{x}^{2}-10 S^{2} S_{x x}+6 S^{5}\right)+ \\
+\alpha_{1}\left(S_{x x}-2 S^{3}\right)+2 x \alpha S+\alpha-4 \delta=0 .
\end{gathered}
$$

Бирациональные преобразования для второй иерархии Пенлеве были приведены в работах [2], [21], [22]. Можно ожидать, что имеется простое обобщение этих преобразований для иерархии (3.29).

Покажем, что уравнения иерархии (3.29) имеют некоторые частные решения. Для этой цели сформулируем следующую теорему. 
ТЕОРема 3.2. Частные решения уравнения (3.29) при $\delta=0$ выражаются через общие решения уравнения (3.12).

ДОКАЗАТЕЛЬСТвО. Уравнение (3.29) при $\delta=0$ можно записать в виде

$$
\begin{gathered}
\left(\frac{d}{d x}-2 S\right) \sum_{k=1}^{m} \alpha_{k} L^{k}\left[S_{x}+S^{2}\right]+2 \alpha x S-\alpha= \\
=\left(\frac{d}{d x}-2 S\right)\left(\sum_{k=1}^{m} \alpha_{k} L^{k}[y]-\alpha x\right),
\end{gathered}
$$

где мы обозначили

$$
y=S_{x}+S^{2}
$$

Из соотношения (3.32) видно, что имеются частные решения уравнений (3.29) при $\delta=0$, которые выражаются через обшие решения уравнений (3.12) при $m=n+1$ с учетом уравнения (3.33). Последнее уравнение можно преобразовать к линейному уравнению

$$
\varphi_{x x}=y \varphi
$$

если предположить, что $S=\varphi_{x} / \varphi$ в $(3.33)$.

Теперь найдем бирациональные преобразования для решений уравнений (3.29). Для этой цели воспользуемся подходом, предложенным в работе [18].

Уравнения (3.29) можно записать, используя две эквивалентные формы:

$$
\begin{array}{r}
\left(\frac{d}{d x}-2 S\right) \sum_{k=1}^{m} \alpha_{k} L^{k}\left[S_{x}+S^{2}\right]+2 \alpha x S-\alpha-4 \delta=0, \\
-\left(\frac{d}{d x}+2 S\right) \sum_{k=1}^{m} \alpha_{k} L^{k}\left[-S_{x}+S^{2}\right]+2 \alpha x S-\alpha=-4 \delta=0 .
\end{array}
$$

Последнее уравнение было найдено с учетом симметрии оператора $L^{k}\left[S_{x}+S^{2}\right]$. Обозначим

$$
\begin{aligned}
& M=\sum_{k=1}^{m} \alpha_{k} L^{k}\left[S_{x}+S^{2}\right]-\alpha x, \\
& N=\sum_{k=1}^{m} \alpha_{k} L^{k}\left[-S_{x}+S^{2}\right]-\alpha x .
\end{aligned}
$$

Тогда уравнения (3.35) и (3.36) можно представить в виде

$$
\begin{gathered}
M_{x}-2 S M-4 \delta=0, \\
N_{x}+2 S^{\prime} N+2 \alpha+4 \delta^{\prime}=0,
\end{gathered}
$$


где мы учли, что

$$
S \equiv S(x, \alpha+4 \delta), \quad S^{\prime} \equiv S^{\prime}\left(x, \alpha+4 \delta^{\prime}\right)
$$

Они представляют собой решения уравнений (3.35) и (3.36).

Используя уравнения (3.39) и (3.40), получим

$$
S=\frac{M_{x}-4 \delta}{2 M}, \quad S^{\prime}=-\frac{N_{x}+2 \alpha+4 \delta^{\prime}}{2 N}
$$

Подставляя $S$ и $S^{\prime}$ в (3.37) и (3.38), можно видеть, что эти уравнения совпадают при $M=N$ в двух случаях:

$$
2 \delta^{\prime}+\alpha=-2 \delta, \quad 2 \delta^{\prime}+\alpha=2 \delta
$$

Первое уравнение (3.42) соответствует симметрии уравнений (3.29) $S \rightarrow-S$ и $4 \delta+$ $\alpha \rightarrow-4 \delta-\alpha$, однако второе уравнение (3.42) приводит к уравнению, которое можно найти из (3.41) при $M=N$ :

$$
S+\frac{\delta}{M}=-S^{\prime}-\frac{\alpha+2 \delta^{\prime}}{M}
$$

Последнее уравнение можно записать в виде бирационального преобразования:

$$
S(x, 4 \delta-\alpha)=-S(x, \alpha+4 \delta)-\frac{4 \delta}{\sum_{k=1}^{m} \alpha_{k} L^{k}\left[S_{x}+S^{2}\right]-\alpha x} .
$$

Эту формулу можно использовать для нахождения рациональных решений уравнений (3.29). Можно видеть, что имеется тривиальное решение этих уравнений $S=0$ при $\delta=-\alpha / 4$. С помошью $(3.44)$, используя $S=0$ в правой части при $\delta=-\alpha / 4$, получим

$$
S(x,-2 \alpha)=-\frac{1}{x}
$$

и т.д. Например, для уравнения (3.30) имеем

$$
\begin{gathered}
S(x,-4 \alpha)=-\frac{2\left(\alpha_{1}+\alpha x^{3}\right)}{x\left(\alpha x^{3}-2 \alpha_{1}\right)} \\
S(x,-6 \alpha)=-\frac{3\left(40 \alpha_{1}^{2} x^{2}-4 \alpha \alpha_{1} x^{5}-48 \alpha_{2} \alpha_{1}-48 \alpha_{2} \alpha x^{3}\right)}{\left(\alpha x^{3}-2 \alpha_{1}\right)\left(\alpha^{2} x^{6}-10 \alpha \alpha_{1} x^{3}+72 \alpha_{2} \alpha x-20 \alpha_{1}^{2}\right)} .
\end{gathered}
$$

Эти решения являются обобшениями решений второго уравнения Пенлеве и второго члена $P_{2}$-иерархии. Решения для $S(x, 2 \alpha), S(x, 4 \alpha)$ и $S(x, 6 \alpha)$ могут быть получены из решений (3.45)-(3.47), если учесть симметрию уравнений (3.29). 
3.3. Случай $\omega(\lambda)=\omega_{0} \lambda^{2}$. Подставляя $\omega(\lambda)=\omega_{0} \lambda^{2}$ и $(2.5)$ в (2.4), получаем

$$
4(P-\lambda) A_{x}+2 A P_{x}-A_{x x x}+\omega_{0} \lambda^{2}=0 .
$$

Полагая $A(x, \lambda)$ имеющим вид $(2.13)$, имеем $a_{0}=c_{0}$ и

$$
a_{k}(x)=\frac{1}{2} \sum_{i=1}^{k}\left(-\frac{1}{4}\right)^{i-1} c_{k-i} L^{i}[P]+c_{k}, \quad k=1, \ldots, n-2 .
$$

Дополнительно получаем также три уравнения

$$
\begin{gathered}
4 P a_{n-2, x}+2 P_{x} a_{n-2}-a_{n-2, x x x}-4 a_{n-1, x}+\omega_{0}=0, \\
4 P a_{n-1, x}+2 P_{x} a_{n-1}-a_{n-1, x x x}-4 a_{n, x}=0, \\
4 P a_{n, x}+2 P_{x} a_{n, x}-a_{n, x x x}=0 .
\end{gathered}
$$

Используя уравнения (3.49), имеем также интеграл вида

$$
\frac{1}{2} \sum_{i=1}^{n-1}\left(-\frac{1}{4}\right)^{i-1} c_{n-1-i} L^{i}[P]+c_{n-1}=-\omega_{0} x
$$

из уравнения (3.50).

С использованием (2.10) можно найти два интеграла уравнений (3.51) и (3.52). Они имеют вид

$$
\begin{gathered}
a_{n, x x} a_{n-1}+a_{n-1, x x} a_{n}+2 a_{n}^{2}-a_{n, x} a_{n-1, x}-4 P a_{n} a_{n-1}+c_{n}=0, \\
a_{n} a_{n, x x}-\frac{1}{2} a_{n, x}^{2}-2 P a_{n}^{2}+c_{n+1}=0 .
\end{gathered}
$$

Обозначая

$$
\begin{gathered}
P(x)=y(x), \quad a_{n-1}(x)=v(x), \quad a_{n}(x)=u(x), \quad \omega_{0}=-\alpha, \\
\alpha_{i}=\frac{1}{2}\left(-\frac{1}{4}\right)^{i-1} c_{n-1-i}, \quad i=1, \ldots, n-2, \quad c_{n}=\chi, \quad c_{n+1}=-\frac{\delta^{2}}{2},
\end{gathered}
$$

из уравнений (3.53)-(3.55) имеем систему уравнений вида

$$
\begin{gathered}
\sum_{i=1}^{n-1} \alpha_{i} L^{i}[y]+4 v(x)=\alpha x, \\
u_{x x} v+v_{x x} u+2 u^{2}-u_{x} v_{x}-4 y u v+\chi=0, \\
u u_{x x}-\frac{1}{2} u_{x}^{2}-2 y u^{2}-\frac{\delta^{2}}{2}=0 .
\end{gathered}
$$

При $n=3$ из (3.57) получаем следующее уравнение:

$$
\alpha_{2}\left(y_{x x}-3 y^{2}\right)+\alpha_{1} y+4 v=\alpha x .
$$

4 Теоретическая и математическая физика, т. 137, № 3, 2003 г. 
Полагая $\alpha_{i}=0, \quad i=1, \ldots, n-1$, в $(3.57)$, имеем $v(x)=\alpha x / 4$. Тогда из уравнений (3.58) и (3.59) получаем частный случай третьего уравнения Пенлеве в виде

$$
u_{x x}-\frac{u_{x}^{2}}{u}+\frac{u_{x}}{x}-\frac{8 u^{2}+4 \nu}{\alpha x}-\frac{2 \delta^{2}}{u}=0 .
$$

Однако, если взять $u(x)=v(x)=\delta=\chi=0$, то из уравнения (3.57) получаем обобщение первой иерархии Пенлеве (3.12). Можно видеть, что систему уравнений (3.57)(3.59) можно рассматривать как обобщение $P_{1}$ - и $P_{3}^{*}$-уравнений.

Очевидно, что систему уравнений (3.57)-(3.59) можно редуцировать к одному уравнению. Из (3.59) имеем

$$
y=\frac{u_{x x}}{2 u}-\frac{u_{x}^{2}}{4 u^{2}}-\frac{\delta^{2}}{4 u^{2}} .
$$

Используя (3.57) и (3.62), получаем

$$
\sum_{i=1}^{n-1} \alpha_{i} L^{i}\left[\frac{u_{x x}}{2 u}-\frac{u_{x}^{2}}{4 u^{2}}-\frac{\delta^{2}}{4 u^{2}}\right]+4 v=\alpha x .
$$

Используя новую функцию $F=\ln u$ в (3.62), имеем

$$
y=\frac{1}{2}\left(F_{x x}+\frac{1}{2} F_{x}^{2}-\frac{\delta^{2}}{2} e^{2 F}\right) .
$$

Подставляя $F=\ln u$ в (3.63), получаем уравнение в виде

$$
\sum_{k=1}^{m} \alpha_{k} L^{k}\left[\frac{1}{2}\left(F_{x x}+\frac{1}{2} F_{x}^{2}-\frac{\delta^{2}}{2} e^{-2 F}\right)\right]+4 v=\alpha x .
$$

Из уравнения (3.58) имеем также

$$
\frac{d}{d x}\left(\frac{d}{d x}-F_{x}\right) v+\delta^{2} v e^{-2 F}+\chi e^{-F}+2 e^{F}=0 .
$$

Последнее можно записать в виде иерархии, если учесть $v(x)$ в уравнении (3.65). Мы получаем

$$
\begin{aligned}
& \frac{d}{d x}\left(\frac{d}{d x}-F_{x}\right) \sum_{k=1}^{m} \alpha_{k} L^{k}\left[\frac{1}{2}\left(F_{x x}+\frac{1}{2} F_{x}^{2}-\frac{\delta^{2}}{2} e^{-2 F}\right)\right]+ \\
& \quad+\delta^{2} e^{-2 F} \sum_{k=1}^{m} \alpha_{k} L^{k}\left[\frac{1}{2}\left(F_{x x}+\frac{1}{2} F_{x}^{2}-\frac{\delta^{2}}{2} e^{-2 F}\right)\right]+ \\
& \quad+\frac{d}{d x}\left(\alpha x F_{x}\right)-\alpha \delta^{2} x e^{-2 F}-4 \chi e^{-F}+\mu e^{F}=0
\end{aligned}
$$

(параметр $\mu$ можно ввести в уравнение (3.67)). Иерархия (3.67) является обобщением иерархии (3.29) согласно следуюшей теореме. 
ТЕОРЕМА 3.3. Частные решения уравнения (3.67) при $m \geqslant 2$ вырахсаются через общие решения $P_{1}-, P_{2}-$ и $P_{3}^{*}$-уравнений.

ДокАЗАТЕльство. Уравнение (3.67) эквивалентно системе уравнений (3.57)-(3.59). Полагая $u=v=\delta=0$, имеем первое уравнение Пенлеве при $\alpha_{k}=0(k>3)$. Поэтому частное решение иерархии (3.67) можно найти, используя обшее решение $P_{1}$-уравнения.

Полагая $\delta=\chi=\mu=0$ и $f(x)=F_{x}$, получаем из уравнения (3.67) при $\alpha_{k}=0(k \geqslant 2)$ после интегрирования

$$
\left(\frac{d}{d x}-f\right)\left(f_{x}+\frac{1}{2} f^{2}\right)+2 \alpha x f-\beta=0
$$

(здесь $\beta$ - постоянная интегрирования). Последнее уравнение является вторым уравнением Пенлеве. Мы получаем, что частное решение уравнения (3.67) также выражается через общее решение $P_{2}$-уравнения.

Полагая $\alpha_{k}=0, k=1, \ldots, m$, в уравнении $(3.67)$, имеем $P_{3}^{*}$-уравнение в виде

$$
\frac{d}{d x}\left(\alpha x F_{x}\right)-\alpha \delta^{2} x e^{-2 F}-4 \chi e^{-F}+\mu e^{F}=0 .
$$

Последнее можно преобразовать к каноническому виду $P_{3}^{*}$-уравнения, если использовать преобразование $F=\ln u$ :

$$
u_{x x}=\frac{u_{x}^{2}}{u}-\frac{u_{x}}{x}+\frac{\delta^{2}}{u}-\frac{\mu u^{2}}{\alpha x}+\frac{4 \chi}{\alpha x} .
$$

Можно видеть, что уравнение (3.67) имеет также частное решение в виде обшего решения уравнения (3.70).

СлЕДСТВИЕ 3.1. $P_{1^{-}}, P_{2^{-}}$и $P_{3}^{*}$-уравнения являются частными случаями иерархии (3.67).

Имеется ОДУ шестого порядка, которое обобшает $P_{1^{-}}, P_{2^{-}}$и $P_{3}^{*}$-уравнения. Оно будет приведено ниже.

Изомонодромийная линейная задача для решения системы уравнений (3.57)-(3.59) снова имеет вид уравнений (2.1).

3.4. Случай $\omega(\lambda)=\omega_{0} \lambda\left(\lambda-\lambda_{0}\right)$. Подстановки $\omega(\lambda)=\omega_{0} \lambda\left(\lambda-\lambda_{0}\right)$ и потенциала (2.5) в уравнение (2.4) приводят к уравнению

$$
A_{x x x}-2 P_{x} A+4(\lambda-P) A_{x}+\omega_{0} \lambda \lambda_{0}-\omega_{0} \lambda^{2}=0 .
$$

Подставляя (2.13) в (3.71) и приравнивая нулю выражения при различных степенях $\lambda$, получаем систему уравнений.

Мы имеем $a_{0}=c_{0}$ и $a_{k}(x), k=1, \ldots, n-2$, в виде (3.49). Дополнительно к этим уравнениям есть уравнения (3.50) и (3.52) и уравнение вида

$$
a_{n-1, x x x}-4 P a_{n-1, x}-2 P_{x} a_{n-1}+4 a_{n, x}+\omega_{0} \lambda_{0}=0 .
$$


Интеграл, соответствующий уравнению (3.72), можно найти по аналогии с уравнением (3.54) из уравнения (2.10) при $\lambda=\lambda_{0}$.

Можно видеть, что в данном случае получается система уравнений, аналогичная уравнениям (3.57)-(3.59). Два уравнения совпадают с уравнениями (3.57) и (3.59), но вместо уравнения (3.58) мы имеем более общее уравнение.

Приведем теперь систему уравнений при $n=3$. Обозначая $P(x)=y(x), a_{3}(x)=$ $u(x), a_{2}(x)=v(x), c_{0}=2 \alpha_{4}, c_{1}=\alpha_{3}, c_{2}=0, c_{3}=\alpha_{1}, \lambda_{0}=\beta, c_{4}=16 \delta$, получаем следуюшую систему уравнений:

$$
\begin{gathered}
\alpha_{4}\left(y_{x x}-3 y^{2}\right)-2 \alpha_{3} y+4 v=\alpha x \\
u_{x x} v+u v_{x x}+2 u^{2}-u_{x} v_{x}-4 y u v+ \\
+\beta\left(v v_{x x}+4 u v-2 y v^{2}-\frac{1}{2} v_{x}^{2}+\alpha_{3}\left(u_{x x}-4 u y\right)+\right. \\
\left.+\alpha_{4}\left(u y_{x x}+y u_{x x}-u_{x} y_{x}-4 u y^{2}\right)\right)+ \\
+\beta^{2}\left(2 v^{2}+\alpha_{3}\left(v_{x x}+4 u-4 y v\right)+\right. \\
\left.+\alpha_{4}\left(v y_{x x}+y v_{x x}-4 u y-v_{x} y_{x}-4 v y^{2}+2 u_{x x}\right)\right)+ \\
+\beta^{3}\left(2 \alpha_{3}\left(2 v-\alpha_{3} y\right)+\alpha_{3} \alpha_{4}\left(y_{x x}-4 y^{2}\right)+\right. \\
\left.+2 \alpha_{4}\left(v_{x x}+4 u-2 y v\right)+\alpha_{4}^{2}\left(y y_{x x}-2 y^{3}-\frac{1}{2} y_{x}^{2}\right)\right)+ \\
+2 \beta^{4}\left(4 \alpha_{4} v-2 \alpha_{3} \alpha_{4} y+\alpha_{4}^{2}\left(y_{x x}-3 y^{2}\right)\right)+\alpha_{1}=0, \\
u u_{x x}-\frac{1}{2} u_{x}^{2}-2 y u^{2}+\delta=0 .
\end{gathered}
$$

Очевидно, что систему уравнений (3.73)-(3.75) можно записать как ОДУ шестого порядка. Мы можем теперь сформулировать следуюшую теорему.

ТЕОрема 3.4. Частные решения системы уравнений (3.73)-(3.75) выражсаются через общие решения $P_{1-}-P_{2-}, P_{3}^{*}-$ и $P_{5}^{*}$-уравнений.

ДокаЗАтЕльство. Полагая $u=v=\alpha_{1}=\alpha_{3}=\beta=\delta=0, \alpha_{4} \neq 0$, мы имеем $P_{1}$ уравнение из (3.73).

В случае $\delta=\alpha_{3}=\beta=0$, принимая во внимание преобразования (3.23) и (3.27), из уравнений (3.73) и (3.75) получаем $P_{2}$-уравнение. Полагая $\alpha_{4}=\alpha_{3}=\beta$, получаем $P_{3}^{*}$ уравнение в виде $(3.70)$.

Поэтому частные решения системы уравнений выражаются по предыдушей теореме через обшие решения $P_{1^{-}}, P_{2^{-}}$и $P_{3}^{*}$-уравнений.

В случае $\alpha_{4}=\alpha_{3}=0$ имеем уравнение из системы уравнений $(3.73)-(3.75)$ в виде

$$
\begin{aligned}
& \frac{\beta u_{x x}}{u}+\frac{4 u_{x x}}{\alpha x}-\frac{\beta u_{x}^{2}}{2 u^{2}}-\frac{4 u_{x}^{2}}{\alpha x u}+\frac{4 u_{x}}{\alpha x^{2}}- \\
& -\frac{16 \beta u}{\alpha x}-\frac{32 u^{2}}{\alpha^{2} x^{2}}+\frac{8 \delta}{\alpha x u}+\frac{\beta \delta}{u^{2}}+\frac{\beta}{2 x^{2}}-\frac{16 \alpha_{1}}{\alpha^{2} x^{2}}-2 \beta^{2}=0 .
\end{aligned}
$$


Последнее уравнение преобразуется к $P_{5}^{*}$-уравнению.

Полагая $\alpha_{1}=0$ и $\beta=0$ в уравнениях (3.73)-(3.75), получаем ОДУ четвертого порядка вида [5]

$$
\begin{aligned}
& \alpha_{0}\left(u_{x x x x}-4 \frac{u_{x} u_{x x x}}{u}+\frac{21}{2} \frac{u_{x}^{2} u_{x x}}{u^{2}}-3 \frac{u_{x x}^{2}}{u}-\frac{9}{2} \frac{u_{x}^{4}}{u^{3}}-5 \delta^{2} \frac{u_{x}^{2}}{u^{3}}-\right. \\
& \left.-\frac{\delta^{4}}{2 u^{3}}+\frac{5 \delta^{2}}{2} \frac{u_{x x}}{u^{2}}\right)-2 \alpha x u_{x x}-2 \alpha u_{x}+2 \alpha_{2}+2 \alpha x \frac{u_{x}^{2}}{u}+\frac{2 \alpha x \delta^{2}}{u}=0,
\end{aligned}
$$

которое при $\alpha_{0}=1$ было получено в недавней работе [5]. Оно является обобщением $P_{3}^{*}$-уравнения.

СЛЕДСТВИЕ 3.2. $P_{1}, P_{2}, P_{3}^{*}$ и $P_{5}^{*}$ являются частными случаями системы уравнений (3.73)- (3.75).

Теперь видно, как из уравнения (2.4) можно получить другие иерархии.

Структура этих иерархий аналогична представленной выше иерархии (3.57)-(3.59). Эти новые иерархии можно записать в виде системы уравнений.

Можно сформулировать следуюшую теорему.

ТеОрема 3.5. Пусть имеется уравнение (2.4) и пусть $A(x, \lambda)$ дается формулой (2.13); тогда уравнение (2.4) приводит $\kappa n+1$ иерархиям $п р и \omega(\lambda)=\omega_{0} \lambda^{m}$, $m=0,1, \ldots, n$.

ДокАЗАТЕЛЬСтво. Эта теорема доказывается путем вычисления всех возможных иерархий при $\omega(\lambda)=\omega_{0} \lambda^{m}, m=0,1, \ldots, n$. Каждая новая степень $\lambda$ в зависимости $\omega(\lambda)$ позволяет найти дополнительный интеграл из уравнения (2.11).

3.5. Случай $\omega(\lambda)=\omega_{0} \lambda^{3}$. Найдем новую систему уравнений из $(2.4)$ при $\omega(\lambda)=$ $\omega_{0} \lambda^{3}$. В этом случае получаем систему уравнений, аналогичную таковой в случае 3.4 . Дополнительный интеграл имеет вид

$$
\begin{aligned}
& 2 P a_{n-1}^{2}+4 P a_{n} a_{n-2}-a_{n-2} a_{n, x x}-4 a_{n} a_{n-1}- \\
& \quad-a_{n-1} a_{n-1, x x}-a_{n} a_{n-2, x x}+a_{n, x} a_{n-2, x}+\frac{1}{2} a_{n-1, x}^{2}-c_{n-2}=0,
\end{aligned}
$$

где $c_{n-2}$ - постоянная. Используя интегралы $(3.54),(3.55),(3.78)$ и обозначая $P(x)=$ $y(x), a_{n-1}(x)=v(x), a_{n-2}(x)=w(x), c_{n}=\delta, c_{n-1}=\mu, c_{n-2}=\nu$, приходим к системе уравнений вида

$$
\begin{gathered}
\sum_{k=1}^{n-2} \alpha_{k} L^{k}[y]+4 w(x)=\alpha x, \\
2 y v^{2}+4 y u w-w u_{x x}-4 u v-v v_{x x}-u w_{x x}+u_{x} w_{x}+\frac{1}{2} v_{x}^{2}-\nu=0, \\
v u_{x x}+v_{x x} u+2 u^{2}-u_{x} v_{x}-4 y u v-\mu=0, \\
u u_{x x}-\frac{1}{2} u_{x}^{2}-2 y u^{2}-\delta=0 .
\end{gathered}
$$

Можно ожидать, что система уравнений (3.79)-(3.82) является также обобшением некоторых уравнений Пенлеве. 


\section{4. ЗАКЛЮЧЕНИЕ}

Суммируем результаты, представленные в данной работе. Используя условие совместности, соответствующее уравнениям Пенлеве, мы нашли несколько новых иерархий, которые обобщают уравнения Пенлеве. По существу мы рассмотрели две изомонодромийные линейные задачи (2.1) и (2.6), которые можно записать как условие совместности (2.4). Далее мы получили два типа обобшений уравнений Пенлеве, одно из которых соответствует $P_{1^{-}}, P_{34^{-}}, P_{2^{-}}, P_{3}^{*}$ - и $P_{5}^{*}$-уравнениям.

В действительности можно найти обобщения уравнений Пенлеве $\left(P_{2}, P_{4}, P_{3}\right.$ и $\left.P_{5}\right)$, принимая во внимание изомонодромийную линейную задачу (2.6) с квадратичным потенциалом (2.9). Для аналогов четвертого порядка эта задача была решена в [5]. Данньй потенциал также использовался ранее в работе [12] для записи новых иерархий $P_{2}$ и $P_{4}$-уравнений. В общем случае можно ожидать нахождения иерархий для $P_{2^{-}}, P_{4^{-}}, P_{3^{-}}$ и $P_{5}$-уравнений аналогично иерархиям из работ [12], [21]-[23].

Мы заметили, что указанные выше уравнения Пенлеве являются частными случаями найденных иерархий. Особое внимание было уделено нелинейным ОДУ шестого порядка. Мы показали, что имеются нелинейные уравнения шестого порядка, обобшающие $P_{1^{-}}, P_{34^{-}}, P_{2^{-}}, P_{3}^{*}$ - и $P_{5}^{*}$-уравнения.

Ранее при исследовании уравнений Пенлеве считалось, что каждое из них можно включить в соответствуюшую иерархию. В действительности обнаружено, что такая ситуация возможна лишь для первой и второй иерархий Пенлеве. Однако из результатов данной работы можно видеть, что имеются иерархии, обобщающие несколько уравнений Пенлеве. В этом случае трудно говорить о какой-либо конкретной иерархии Пенлеве.

Результаты данной работы позволяют высказать предположение, что имеются нелинейные уравнения высших порядков, включаюшие все уравнения Пенлеве, соответствуюшие конкретной изомонодромийной линейной задаче. Более того, мы полагаем, что имеются обшие решения ОДУ высших порядков в виде трансцендентных функций по отношению к постоянным интегрирования, которые содержат трансценденты Пенлеве определенного класса как частные случаи. Эти общие трансценденты можно найти как решения задач Коши с помошью обратного преобразования монодромии из изомонодромийных линейных задач, соответствуюших ОДУ высших порядков.

Благодарности. Данная работа была поддержана со стороны Международного научно-технического центра (проект 1339-2). Материал данной статьи частично основан на работе, поддержанной грантом РФФИ № 01-01-00693.

\section{Список литературы}

[1] R. Conte. The Painlevé approach to nonlinear ordinary differential equations. In: The Painleve Property. One Century Later. CRM Series in Math. Phys. Ed. R. Conte. New York: Springer, 1999. P. 77-108; M. J. Ablowitz, P. A. Clarkson. Solitons, Nonlinear Evolution Equations and Inverse Scattering. Cambridge: Cambridge Univ. Press, 1991.

[2] H. Airault. Stud. Appl. Math. 1979. V. 61. P. 31.

[3] N. A. Kudryashov. Phys. Lett. A. 1997. V. 224. P. 353. 
[4] N. A. Kudryashov. Phys. Lett. A. 1999. V. 252. P. 173; A. N. W. Hone. Physica D. 1998. V. 118. P. 1; P. R. Gordoa, A. Pickering. J. Math. Phys. 1999. V. 40. P. 5749.

[5] N. A. Kudryashov. J. Phys. A. 2002. V. 35. P. 4617.

[6] R. Garnier. Ann. Ec. Norm. 1912. V. 29. P. 1-126.

[7] H. Flachka, A. C. Newell. Commun. Math. Phys. 1980. V. 76. P. 65.

[8] M. Jimbo, T. Miwa, K. Ueno. Physica D. 1981. V. 2. P. 306.

[9] R. Conte, M. Musette. Chaos, Solitons and Fractals. 2000. V. 11. P. 41.

[10] В. Э. Адлер, А. Б. ШШабат, Р.И. Ямилов. ТМФ. 2000. Т. 125. С. 355.

[11] C. Cresswell, N. Joshi. The discrete Painlevé I hierarchy. In: Symmetries and Integrability of Difference Equations. Proc. of the 2nd Int. Conf. (Caunterbury, UK, July 1-5, 1996). Lond. Math. Soc. Lect. Notes Ser. V. 255. Eds. P.A. Clarkson et al. Cambridge: Cambridge Univ. Press, 1999. P. 197-205.

[12] P. R. Gordoa, N. Joshi, A. Pickering. Publ. RIMS Kyoto Univ. 2001. V. 37. P. 327.

[13] P. D. Lax. Pure Appl. Math. 1968. V. 21. P. 467.

[14] A. Pickering. Phys. Lett. A. 2002. V. 301. P. 275.

[15] C. M. Cosgrove. Stud. Appl. Math. 2000. V. 104. P. 1.

[16] U. Mugan, F. Jrad. J. Phys. A. 1999. V. 32. P. 7933.

[17] N. A. Kudryashov. J. Nonlinear Math. Phys. 2001. V. 8. P. 172.

[18] N. A. Kudryashov, M. B. Soukharev. Phys. Lett. A. 1998. V. 237. P. 206.

[19] В. И. Громак. Дифф. уравнения. 1999. Т. 35. С. 38; P. R. Gordoa. Phys. Lett. А. 2001. V. 287. P. 365; N. A. Kudryashov. J. Phys. A. 1998. V. 31. P. L129; 1999. V. 32. P. 999.

[20] P. A. Clarkson, N. Joshi, A. Pickering. Inverse Problems. 1999. V. 15. P. 175; V. I. Gromak. Bäcklund transformations of Painlevé equations and their applications. In: The Painleve Property. One Century Later. CRM Series in Math. Phys. Ed. R. Conte. New York: Springer, 1999. P. 687-734.

[21] U. Mugan, F. Jrad. J. Nonlinear Math. Phys. 2002. V. 9. P. 1.

[22] A. N. W. Hone. J. Phys. A. 2001. V. 34. P. 2235.

[23] M. Noumi, Y. Yamada. Commun. Math. Phys. 1998. V. 199. P. 281; Funkcial. Ekvac. 1998. V. 41. P. 483. 